\title{
Profil Évolutif Des Accidents Vasculaires Cérébraux Hémorragiques À Yaoundé (Hôpital Général \& Centre Hospitalier Et Universitaire)
}

\author{
Patrice Emmanuel Awono Ateba, Inf. - Epidemiologist, Msc in \\ Public Health \\ Yaoundé General Hospital, Cameroon \\ Justin Ndié, Inf. - Epidemiologist, Msc in Public Health \\ Ministry of Public Health, Cameroon \\ Julienne Louise Ngo Likeng, Anthropologist, PhD, Senior \\ Lecturer \\ School of Health Sciences of Catholic University of Central Africa \\ Benjamin Alexandre Nkoum, Inf., PhD, Senior Lecturer \\ School of Health Sciences of Catholic University of Central Africa, \\ Cameroon
}

doi: 10.19044/esj.2016.v12n21p197 URL:http://dx.doi.org/10.19044/esj.2016.v12n21p197

\begin{abstract}
Nowadays, strokes constitute a real public health problem in the world. In Cameroon, because of the people's lifestyle potential risk factor, the prevalence of hemorrhagic strokes as well as its mortality is on the rise. This study was aimed at analyzing the evolutionary profile of hemorrhagic strokes at the General Hospital and the University Teaching Hospital in Yaoundé. A retrospective cross-sectional study with a qualitative component was carried out at the General Hospital and the University Teaching Hospital in Yaoundé for 2 months. Were included, all patients with a diagnosis of hemorrhagic strokes confirmed by CT Scan. With the help of a scale, patient data over a 30 month's period (2013-2015) were collected. Over 1037 patients hospitalized in intensive care unit at the General Hospital and the University Teaching Hospital in Yaoundé, 122 cases of hemorrhagic strokes were diagnosed, that is a prevalence of $11.8 \%$. The average age was $58 \pm 10.52$ and the most vulnerable age range was between 50 and 70 years with $67.2 \%$. Men were most affected (60.7\%) with a sex ratio of 1.54(74 men/48 women). Among these cases of hemorrhagic strokes still hospitalized, 70 had died giving a lethality rate of $57.4 \%$. Only the duration of hospitalization had an effect on the outcome of patients with hemorrhagic strokes. Actually the patients, victims of hemorrhagic strokes who had had
\end{abstract}


less than 21 days of hospitalization, had 2.91 chances of dying as compared to those patients hospitalized for over 21 days (OR=2.91 IC 95\% [5.1-65.7]; $\mathrm{p}=0.000$ ). The late admission to reanimation, the difficulties encountered by victims of hemorrhagic strokes and their careers, to handle the enormous cost of their treatment and the insufficiencies of the technical platform, were factors also influencing the evolution of hemorrhagic strokes hospitalized in reanimation at the General Hospital and the University Teaching Hospital in Yaoundé. Despite certain efforts aimed at reducing premature mortality, the prevalence of hemorrhagic strokes was increasing in reanimation at the General Hospital and University Teaching Hospital in Yaoundé. Only multisectorial actions of sensitization, the restructuring and amelioration of the care of this ailment will help reverse the evolutionary trend.

Keywords: Evolutionary profile, strokes, hemorrhagic strokes, General Hospital Yaoundé, University Teaching Hospital Yaoundé

\section{Résumé}

De nos jours, les accidents vasculaires cérébraux constituent un véritable problème de santé publique dans le monde. Au Cameroun, à cause du mode de vie des populations, potentiel facteur de risque, la prévalence des accidents vasculaires cérébraux hémorragiques ainsi que sa mortalité sont en nette progression. Cette étude vise à analyser le profil évolutif des accidents vasculaires cérébraux hémorragiques à l'Hôpital Général et au Centre Hospitalier Universitaire de Yaoundé. Une étude transversale rétrospective couplée à un volet qualitatif a été menée à l’Hôpital Général et au Centre Hospitalier et Universitaire de Yaoundé sur 2 mois. Était inclus, tout patient avec un diagnostic d'accident vasculaire cérébral hémorragique confirmé au scanner. À l'aide d'une grille, les données des patients sur une période de 30 mois (janvier 2013 - juin 2015) ont été collectées. Sur 1037 patients hospitalisés en réanimation à l'Hôpital général et au Centre Hospitalier Universitaire de Yaoundé, 122 cas d'accidents vasculaires hémorragiques ont été diagnostiqués soit une prévalence hospitalière de 11,8\%. Les hommes étaient majoritairement atteints (60,7\%) avec un sex-ratio de 1,54 (74H/48F). L'âge moyen était de $58 \pm 10,52$ et la tranche d'âge la plus touchée était celle de 50 à 70 ans avec 67,2\%. De ces cas hospitalisés pour accidents vasculaires cérébraux hémorragiques, 70 étaient décédés soit un taux de létalité de 57,4\%. Seule la durée d'hospitalisation avait un effet sur le devenir des patients victimes d'AVC hémorragiques $(p=0,000)$. En effet, les patients qui avaient eu une durée d'hospitalisation inférieure à 21 jours avaient 2,91 fois plus de chance de décéder que les patients qui avaient séjourné plus de 21 jours (OR= 2,91 IC95\% [5,1 - 65,7] ; p=0,000). De plus, le délai d'admission tardif en réanimation, la difficulté pour les 
victimes des accidents vasculaires cérébraux hémorragiques et/ou leurs accompagnants de couvrir les énormes frais de leur prise en charge et les insuffisances du plateau technique avaient été énoncés, comme des facteurs influençant aussi l'évolution des accidents vasculaires cérébraux hémorragiques dans ces formations sanitaires. Malgré des efforts en faveur de la réduction de la mortalité précoce, la prévalence d'accidents vasculaires cérébraux hémorragiques était en progression en réanimation à l'Hôpital Général et au Centre Hospitalier Universitaire de Yaoundé. Seules des actions multisectorielles de sensibilisation, la restructuration et l'amélioration de la prise en soins de cette affection devraient aider à inverser la tendance de l'évolution et sa mortalité.

Mots-clés : Profil évolutif, accident vasculaire cérébral hémorragique, Hôpital Général de Yaoundé, Centre Hospitalier et Universitaire de Yaoundé

\section{Introduction}

Les accidents vasculaires cérébraux (AVC) et précisément les accidents hémorragiques sont une cause importante de handicap et de mortalité dans le monde. Ils demeurent un problème de santé publique dans les pays en développement où le nombre de cas est en nette progression avec le cours de la transition épidémiologique. L’hypertension artérielle reste le principal facteur de risque des accidents vasculaires cérébraux (Goldstein et al., 2011 ; O'Donnell et al., 2010 ; Touzé \& Bejot, 2009). En effet, selon Bejot et al. (2009b), chaque augmentation de la pression artérielle systolique de $20 \mathrm{mmHg}$ ou de la pression artérielle diastolique de $10 \mathrm{mmHg}$ est associée à un doublement du risque d'AVC quel que soit l'âge. En outre, la race, l'obésité, le diabète, les habitudes alimentaires et bien d'autres éléments socio-économiques sont des facteurs dits secondaires ou associés au risque d'AVC hémorragiques (Ndoumba et al., 2012 ; Hu et al., 2007 ; Schneider et al., 2007 ; Goldstein et al., 2006 ; Johnsen, 2004 ; Reynolds, 2003 ; He et al., 2002).

Les accidents vasculaires cérébraux demeurent la première cause de handicap chez les adultes (HAS, 2009). Chaque année, près de 15 millions de personnes dans le monde sont victimes d'un AVC et près de 5 millions en meurent (OMS, 2005). En outre, 10\% de la mortalité mondiale est imputée aux accidents vasculaires cérébraux (Ibid.). Toutefois, si aucune disposition n'était prise, le monde devrait connaître 18 millions de nouveaux cas d'AVC et 6,5 millions de décès par AVC en 2015 (Mensah, 2008 ; Strong et al., 2007). Par ailleurs, ils estimaient à 42 millions de nouveaux cas d'AVC et 7,8 millions de décès attendus d'ici 2030 si les tendances et les conditions actuelles persistaient. 
En 2012, l’OMS affirmait que 4/5 des décès dus aux AVC se dénombre dans les pays pauvres, avec une tendance à l'augmentation (OMS, 2012). Par ailleurs, Callow (2006) remarquait que les maladies cardiovasculaires étaient en constante progression de façon particulière en Afrique de 15\% en 1995 à 35\% en prévision pour l'année 2015. Pour Kengne et Anderson (2006), en Afrique sub-saharienne, la prévalence des AVC serait autour de 200 à 300 pour 100000 personnes avec une augmentation de la charge de la maladie de près de $400 \%$ ces deux dernières décennies (Ibid.). Cependant, la mortalité des AVC en Afrique reste supérieure à celle de la plupart des pays à hauts revenus (Strong et al. 2007). Au Sénégal, les AVC constituent plus de 30\% des hospitalisations et sont responsables de 2/3 de la mortalité dans le service de neurologie (Sene Diouf, 2006).

$\mathrm{Au}$ Cameroun, les maladies chroniques non transmissibles restent négligées dans les programmes de santé bien qu'étant la première cause de décès et d'invalidités (MINSANTE, 2011). En effet, l'OMS (2011), estimait la prévalence des maladies cardiovasculaires à 14\% dans ce pays. Partant, les maladies cardiovasculaires progressant, entraînent de fait la hausse du nombre d'AVC. Selon Ndoumba et al. (2012), le taux d'hospitalisation tourne autour 10,4\% dans les centres de soins. Par ailleurs, Tossou (2014) notait une progression significative des cas à l'Hôpital Central de Yaoundé passant de 706 cas en 2010 à 1534 cas en 2013. En 2012, la mortalité intra hospitalière due aux AVC était estimée à 13.6\% et se situait dans les deux premières semaines d'hospitalisation (Gantchou Nana, 2012). Cependant, dans les formations sanitaires de Yaoundé, le plateau technique reste faible et les patients peinent à couvrir les coûts relatifs à la prise en soins. Cette situation contribue davantage à accroître la mortalité amputable aux AVC. La présente étude vise à analyser le profil évolutif des accidents vasculaires cérébraux hémorragiques à l’Hôpital général (HGY) et au Centre Hospitalier Universitaire de Yaoundé (CHUY).

\section{Matériels et méthodes}

\section{Cadre de l'étude}

Deux structures de $4^{\text {ème }}$ référence (niveau de référence le plus élevé au Cameroun) ; en l'occurrence l'Hôpital Général et le Centre Hospitalier et Universitaire de Yaoundé au Cameroun, pays d'Afrique centrale ont servi de cadre à l'enquête de la présente étude. Ces structures sanitaires de référence de Yaoundé (capitale du Cameroun), sont dotées d'unités de soins intensifs, service le plus approprié pour manager les cas d'AVC hémorragique. En outre, elles sont censées avoir le meilleur plateau technique et par conséquent, devraient fournir l'offre de soins la plus adaptée en matière de prise en charge des AVC. Aussi, leur niveau sur la pyramide sanitaire et leur 
situation géographique leur permet d’accueillir les patients venant de tous les horizons du pays.

\section{Méthodologie}

Une étude transversale rétrospective couplée à un volet qualitatif a été réalisée à l’Hôpital Général et au Centre Hospitalier et Universitaire de Yaoundé au Cameroun. Était inclus, tout patient avec un diagnostic d’accident vasculaire cérébral hémorragique confirmé au scanner ainsi que les responsables des unités de soins intensifs de ces formations sanitaires. À l'aide d'une grille, nous avons compulsé les dossiers des patients internés en réanimation dans ces hôpitaux sur une période de 30 mois (janvier 2013 juin 2015). La variable dépendante de cette étude était le devenir des patients (sorti, décédé). Les variables explicatives étaient les variables sociodémographiques (sexe, âge, profession, lieu de résidence des patients) et les variables liées à l'évolution (année, lieu d'hospitalisation et la durée d'hospitalisation). La saisie, le traitement et l'analyse des données ont été réalisées à l'aide des logiciels SPSS et Excel.

Le test de khi 2 a permis de mesurer l'association entre les variables au seuil de significativité à 5\% et le $\mathrm{V}$ de Cramer variant entre [0-1] a permis de mesurer la force de l'association. La régression logistique binaire a permis de mesurer l'effet de certaines variables sur le devenir des patients victimes d'AVC hémorragiques.

À l'aide d'un guide d'entretien, des entretiens ont été menés avec 4 responsables (informateurs clés) de ces centres : 2 médecins (responsables d'unité de soins intensifs) et 2 infirmiers (Majors de soins intensifs). Après avoir transcrit manuellement les données, nous avons procédé à l'analyse de contenu et à un regroupement des données en items.

\section{Résultats}

\section{Analyse des données quantitatives}

Entre janvier 2013 et juin 2015, sur 1037 hospitalisés dans ces centres de références, 122 cas d’AVC hémorragiques confirmés par un scanner cérébral ont été recensé représentant une prévalence hospitalière de 11,8\%. La majorité des cas (64,8\%), avaient été diagnostiqués au CHUY.

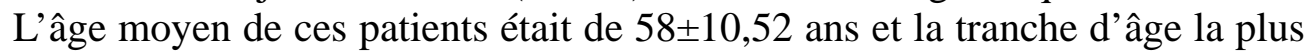
touchée était celle de 50 à 70 ans avec 67,2\%. Les hommes étaient majoritairement atteints (60,7\%), avec un sex-ratio de 1,54 (74H/48F). Les personnes n’exerçant aucune activité génératrice de revenu étaient les plus touchées $(47,5 \%)$ suivi des personnes exerçant dans le secteur libéral $(29,5 \%)$ et enfin des fonctionnaires (23\%). Une part importante de ces patients (39,3\%), avait passé un court séjour dans le centre soit moins de 5 jours d’hospitalisation. La majorité des patients était décédé soit un taux de 
létalité de 57,4\%.

Il existait une association significative entre l'âge des patients victimes d'AVC hémorragiques et leur devenir. En effet, l'âge moyen des patients victimes d'AVC hémorragiques différait significativement $(p=0,046)$ selon leur devenir (56,38 chez les patients sortis ; 60,21 chez les décédés).

Tableau 1 : Association entre les facteurs et le devenir des patients

\begin{tabular}{|c|c|c|c|c|}
\hline \multirow{3}{*}{ Formation sanitaire } & \multicolumn{2}{|c|}{ Devenir du patient } & \multirow[b]{2}{*}{$\mathrm{X}^{2}$} & \multirow[b]{2}{*}{ p-value } \\
\hline & Décédé n(\%) & Sorti n(\%) & & \\
\hline & & & & \multirow{3}{*}{0.031} \\
\hline CHUY & $40(57,1)$ & $39(75)$ & \multirow{2}{*}{4,17} & \\
\hline HGY & $30(42,9)$ & $13(25)$ & & \\
\hline \multicolumn{5}{|c|}{ Activité génératrice de revenu } \\
\hline Non & $39(55,7)$ & $19(36,5)$ & \multirow{2}{*}{4,4} & \multirow{2}{*}{$\mathbf{0 , 0 2 7}$} \\
\hline Oui & $31(44,3)$ & $33(63,5)$ & & \\
\hline \multicolumn{5}{|l|}{ Durée d'hospitalisation } \\
\hline$<5$ jours & $42(60)$ & $6(11,5)$ & \multirow{3}{*}{32,4} & \multirow{3}{*}{0.000} \\
\hline$[5-20]$ jours & 21(30) & $25(48,1)$ & & \\
\hline Plus de 20 jours & $7(10)$ & $21(40,4)$ & & \\
\hline
\end{tabular}

De ce tableau, il ressort que le devenir des patients victimes d'AVC hémorragiques était statistiquement associé à la formation sanitaire $\left(\mathrm{X}^{2}=\right.$ $4,17 ; \mathrm{p}=0,031)$; l'activité génératrice de revenu $\left(\mathrm{X}^{2}=4,4 ; \mathrm{p}=0,027\right)$ et à la durée d'hospitalisation $\left(X^{2}=32,4 ; p=0,000\right)$.

En analyse multivariée (régression logistique binaire), seule la durée d'hospitalisation avait un effet sur le devenir des patients victimes d'AVC hémorragiques dans ces centres $(p=0,000)$. En effet, les patients victimes d'AVC hémorragiques qui avaient eu une durée d'hospitalisation inférieure à 21 jours, avaient 2,91 fois plus de chance de décéder que les patients qui avaient séjourné plus de 21 jours ( $\mathrm{OR}=2,91 \mathrm{IC} 95 \%[5,1-65,7] ; \mathrm{p}=0,000)$.

\section{Analyse des données qualitatives} À propos des ressources

La disponibilité des ressources humaines mais surtout matérielles dans les centres de soins permet d'améliorer la performance. À cet effet, les informateurs déclarent :

"Le matériel de réanimation est inadéquat et insuffisant, par rapport au nombre de cas. Les produits appropriés manquent souvent et cela nous empêche de bien nous occuper des malades souffrant d'AVC» (Informateur 1, responsable de l'unité des soins intensifs, CHUY).

"Le service de réa connaît d'énormes de problème de matériels et de personnels alors qu'il faut vite agir sur les malades » (Informateur 3, responsable de l'unité des soins intensifs, HGY). 
"Les appareils de surveillance continue comme les moniteurs, la disponibilité permanente de l'oxygène mural et d'autres appareils appropriés de réanimation tels les aspirateurs, la ventilation artificielle, nous permettent d'avoir souvent de bon résultats» (Informateur 2, responsable des soins, CHUY).

\section{À propos du coût de la prise en charge}

La majorité des informateurs clés s’accordaient sur le fait que la prise en charge des AVC et les coûts associés sont exorbitants et ne sont pas à la portée du citoyen ordinaire.

"L'hospitalisation en réanimation pour les AVC n'est pas donnée, il faut compter dans les un million cinq cent mille francs CFA pour les trois semaines à un mois d'hospitalisation qui sont le minimum exigé» (Informateur 2, responsable des soins, CHUY).

«... d'ailleurs plusieurs familles, quand on leur parle d'une caution de deux cent à trois cent mille, elles repartent souvent avec leurs malades. Pour ceux qui restent, très peu arrivent à couvrir les besoins financiers de l'hospitalisation et on a ainsi un bon nombre de familles qui signent la sortie exigée avant même que le patient ne soit complètement sorti d'affaire, certains en cours d'hospitalisation n'arrivent plus à acheter tous les médicaments » (Informateur 4, responsable des soins, HGY).

\section{Concernant l'évolution de la maladie}

Compte tenu des insuffisances du plateau technique et de la non subvention des coûts relatifs à la prise en soins des AVC, tous les informateurs reconnaissaient que le pronostic des AVC est couramment défavorable.

"Ceux qui n'ont pas assez de moyens et ceux qui arrive avec d'autres pathologies, s'en sortent moins bien que les autres» (Informateur 3, responsable de l'unité des soins intensifs, HGY).

"La situation ici est très grave. Presque tous nos cas d'AVC hémorragiques meurent; nous on est même un peu découragés » (Informateur 4, responsable des soins, HGY).

En somme, le délai d'admission tardif en réanimation, la difficulté pour les victimes des accidents vasculaires cérébraux hémorragiques et leurs accompagnants ; de couvrir les énormes frais de leur prise en charge et les insuffisances du plateau technique, avaient été énoncés par les informateurs clés comme des facteurs influençant aussi l'évolution des accidents vasculaires cérébraux hémorragiques à l'HGY et au CHUY.

\section{Discussion}

La majorité des cas d’AVC (64,8\%), avaient été diagnostiqués et 
hospitalisés au CHUY. Cette fréquence élevée trouve sa justification dans le fait qu'à la période 2013-2015, cette institution avait un plateau technique relativement plus élevé que celui de l'HGY et partant, une meilleure capacité d'accueil des patients victimes d'AVC hémorragiques. D’ailleurs, un responsable de cette institution déclare à cet effet: "les appareils de surveillance continue comme les moniteurs, la disponibilité permanente de l'oxygène mural et d'autres appareil appropriés de réanimation tels les aspirateurs, la ventilation artificielle, nous permettent d'avoir souvent de bon résultats » (Informateur 2, responsable des soins, CHUY).

Les hommes étaient majoritairement atteints par les AVC contrairement aux femmes. Ces résultats sont similaires à ceux de Keita et al. (2005) qui avaient trouvé 56,6\%. Les résultats de Chiasseu Mbeumi et Mbahe (2011) étaient même 1,19 fois supérieurs aux nôtres avec 64\% d'hommes. De plus, Beyiha et al. (2008) avaient trouvé un sex-ratio de 2,3. Ces études relèvent toutes, une prévalence masculine élevée mettant en lumière le fait que les AVC touchent un peu plus les hommes que de femmes. Ainsi, les hommes sont-ils relativement plus enclins aux facteurs de risque d'AVC notamment l'hypertension artérielle, le tabagisme et l'alcoolisme ? Par ailleurs, l'altération des conditions sociales et de travail dont ils sont victimes contribue au maintien ou à l'augmentation du stress, autre facteur de risque des AVC. D'amples études menées sur l'ensemble de la population permettraient d'apprécier le niveau d'influence de ces facteurs sur cette frange de la population. À contrario, Sène Diouf et al. (2006), au Sénégal, avaient trouvé un sex-ratio 0,68 .

L'âge moyen était de $58 \pm 10,52$ ans et la tranche d'âge la plus touchée était celle de 50 à 70 ans avec 67,2\%. Nos résultats sont similaires à ceux de Wafeu Sadeu (2015) à Yaoundé et comparables à ceux de Chiasseu Mbeumi et Mbahe (2011) à Douala qui montraient que la tranche d'âge la plus atteinte est celle comprise entre 51 ans et 60 ans. Par contre, ils étaient nettement supérieurs à ceux de Keita et al. (2005) au Mali et de Beyiha et al. (2008) au Cameroun, qui rapportaient respectivement un âge moyen de 45,5 ans et de 55,5 ans. Cependant, ils étaient légèrement inférieurs à ceux de Sène Diouf et al. (2006) au Sénégal qui avaient trouvé un âge moyen de $61 \pm 13$ ans. Ces résultats laissent percevoir que les AVC touchent essentiellement les individus au-delà de la cinquantaine. En effet, ces données révèlent que c'est autour de la cinquante que surviennent le plus souvent les maladies cardiovasculaires dont l'hypertension artérielle qui est le principal facteur de risque des AVC.

Concernant la durée du séjour, une part importante des patients (39,3\%), avait passé un court séjour dans les centres soit moins de 5 jours d'hospitalisation. Ces résultats sont nettement inférieurs ( 0,75 fois) à ceux de Sène Diouf et al. (2008) qui rapportaient que la durée moyenne du séjour en 
neuro-réanimation à Dakar était de 10,8 \pm 11 jours. Ce bref séjour dans les centres de soins était marqué par une fréquence élevée de décès (60\%), témoignant la faiblesse de la prise en charge précoce. En effet, une prise en charge précoce adéquate permet d'atténuer les effets morbides induits dans les premières heures de l'installation d'un AVC hémorragique. Or dans les formations sanitaires en général et dans les centres de références en particulier, se pose le problème de matériels de pointe des soins intensifs et de ressources humaines qualifiées. De ce point de vue, certains informateurs l'expriment ainsi : "le matériel de réanimation est inadéquat et insuffisant, par rapport au nombre de cas. Les produits appropriés manquent souvent et cela nous empêche de bien nous occuper des malades souffrant d'AVC; le service de réa connaît d'énormes de problème de matériels et de personnels alors qu'il faut vite agir sur les malades » (Informateur 2, responsable des soins, CHUY ; informateur 3, responsable de l'unité des soins intensifs, HGY).

La majorité des patients était décédée soit un taux de létalité de 57,4\%. Sène Diouf et al. (2008) au Sénégal avaient trouvé une mortalité 1,5 fois plus élevée soit 82,9\%. Par contre, Keita et al. (2005), avaient trouvé une mortalité sensiblement inférieure de $45,7 \%$. La mortalité élevée traduit la faiblesse du système de prise en charge précoce dans les centres de référence. À cet effet, un informateur déclare : "La situation ici est très grave. Presque tous nos cas d'AVC meurent; nous on est même un peu découragés » (Informateur 4, responsable des soins, HGY).

En effet, les patients victimes d'AVC hémorragiques ayant séjourné moins de 21 jours en hospitalisation à l'HGY ou au CHUY avaient 2,91 fois plus de chance de décéder que les patients qui avaient séjourné plus de 21 jours $(\mathrm{OR}=2,91 \mathrm{IC} 95 \%$ [5,1-65,7] ; $\mathrm{p}=0,000)$. En d'autres termes, plus un patient avait un séjour long, plus ses chances de sortir vivant du centre étaient grandes. Ainsi, le séjour relativement long d'un patient d'AVC dans un centre de référence (HGY ou CHUY) était statistiquement associé à un risque réduit du décès de celui-ci $(p=0,000)$.

De ces décès, les personnes n’ayant aucune activité génératrice de revenu étaient les plus touchées avec 55,7\%. En effet, les personnes n'ayant aucune activité sont davantage exposées à la sédentarité qui est un facteur de risque des AVC. Cette situation peut expliquer cette prévalence élevée chez cette frange de la population. Par ailleurs, ils éprouvaient plus de difficultés à couvrir les coûts relatifs à leurs soins. Ce non payement de soins s'apparente à une cause sous-jacente de mortalité comme le traduisent ces propos des informateurs : " ceux qui n'ont pas assez de moyens et ceux qui arrivent avec d'autres pathologies, s'en sortent moins bien que les autres ; ... d'ailleurs plusieurs familles, quand on leur parle d'une caution de deux cent à trois cent mille, repartent souvent avec leurs malades. Pour ceux qui restent, très 
peu arrivent à couvrir les besoins financiers de l'hospitalisation et on a ainsi un bon nombre de familles qui signent la sortie exigée avant même que le patient ne soit complètement sorti d'affaire, certains en cours d'hospitalisation n'arrivent plus à acheter tous les médicaments » (Informateur 3, responsable de l'unité des soins intensifs, HGY ; informateur 4, responsable des soins, HGY). Par ailleurs, tous les informateurs étaient unanimes sur le fait, qu'une prise en charge précoce et optimale, la disponibilité des moyens financiers et technique, et la coexistence de l'AVC hémorragique avec d'autres pathologies exerçaient une influence significative sur l'évolution des malades.

\section{Conclusion}

Les maladies cardiovasculaires, au rang desquelles les accidents vasculaires cérébraux hémorragiques restent une menace pour la survie des populations ; constituant de fait un problème de santé publique majeur. Dans les pays en développement en général et au Cameroun en particulier, les AVC sont devenus fréquents. Ils sont responsables d'importantes séquelles et de handicap chez les survivants et en même temps, ils causent une mortalité importante. La prévalence d'accidents vasculaires cérébraux hémorragiques était en progression en réanimation à l’Hôpital Général et au Centre Hospitalier Universitaire de Yaoundé. Le CHUY était le centre qui avait diagnostiqué et hospitalisé la majorité des cas (64,8\%), les hommes (56\%) étaient majoritairement atteints et la létalité globale était de 57,4\%. Cependant, le plateau technique était insuffisant et inadéquat mais aussi, les patients peinaient à couvrir le coût de leur hospitalisation. Ce qui contribuait de façon sous-jacente à augmenter le taux de mortalité. Malgré certains efforts, les AVC hémorragiques restent fréquents. La mise en œuvre effective des programmes de prévention des facteurs de risque au sein des populations par les pouvoirs publics, le renforcement des systèmes de prise en charge dans les différents centres et la subvention des coûts de soins permettront de réduire sensiblement la mortalité imputable aux accidents vasculaires cérébraux. En outre, une enquête à l'échelle nationale permettra de cerner la prévalence de ce fléau.

\section{References:}

Anderson, CS. \& al. (2008). Intensive blood pressure reduction in acute cerebral hemorrhage trial (INTERACT): a randomised pilot trial. Lancet Neurol. 7.

Andrews, PJ., Piper IR. Dearden NM. \& Miller J. D. (1990). Secondary insults during intra hospital transport of head-injured patients. Lancet. 10. Bejot et al. (2009). Épidémiologie des accidents vasculaires cérébraux. Medicine/sciences. 25 : 8-9. 
Beyiha, Q._et al. (2008). Aspects épidémiologiques et facteurs de gravité des accidents vasculaires cérébraux au Cameroun. Journal maghrébin d'anesthésie-réanimation et de médecine d'urgence. 15(66) : 293-297.

Callow (2006). Cardiovascular disease 2005- the global picture. Vascular pharmacology. 45. (5).

Chiasseu Mbeumi, M. T. \& Mbahe, S. (2011). Étude descriptive des accidents vasculaires cérébraux à Douala, Cameroun. Med. Trop. 71 : 492494.

Gantchou Nana, M. (2012). Étude épidémio-clinique et évolutive de l'AVC du sujet jeune en milieu hospitalier au Cameroun, mémoire. Master en Santé Publique. ESS-UCAC.

Goldstein et al. (2006). Primary prevention of ischemic stroke. A guide line from the American heart association stroke council.

Goldstein et al. (2011). Guide line for the primary prevention of stroke: a guideline for healthcare professionals from the American heart association/American stroke Association. Stroke. 42(2).

HAS (2009). Messages clés destinés aux médecins généralistes, AVC : prise en charge précoce. République française.

HAS (2009, Mai). Recommandations de bonne pratique Argumentaire AVC : prise en charge précoce. République française.

He, J. et al. (2002). Fish consumption and risk of stroke in men. JAMA: the journal of the American association. 288(24).

He, J., Whelton PK, Vu B. \& Klag MJ. (1998). Aspirin and risk of hemorrhagic stroke: a meta-analysis of randomized controlled trials. JAMA. 280.

Hu et al. (2007). Body mass index, waist circumference and waist-hip ratio on the risk of total and type of stroke. Archives of internal medicine. 167(13).

Johnsen (2004). Intake of fruit and vegetables and risk of stroke: and overview. Current opinion in clinical and metabolic care. 7(6).

Keita, D. M. et al. (2005). Aspects épidémiologiques des accidents vasculaires cérébraux dans le Service de tomodensitométrie à L’Hôpital du Point G. Med. Trop. 65(71): 453-457.

Kengne A. P. \& Anderson C. (2006). The neglected burden of stroke in SubSaharan Africa. International Journal of stroke. 1.

Kengne A.P., Awah P.K., Fezeu L. \& Mbanya J.C. (2007). The burden of high blood pressure and related risk factors in urban sub Saharan Africa: evidences from Douala in Cameroon. African health sciences. 7.

Mensah (2008). Epidemiology of stroke and high blood pressure in Africa. Heart. 96(6).

MINSANTE (2011). Plan stratégique national de prévention et contrôle des maladies chroniques non transmissible 2011-2015 au Cameroun. Yaoundé. 
République du Cameroun.

Ndoumba Mintya, A. et al. (2012). Les accidents vasculaires et cérébraux à l'hôpital gynéco obstétrique et pédiatrique de Yaoundé; aspects épidémiologiques, cliniques et para cliniques. Schweizer Archiv Für Neurologie Und Psychiatrie. 163.

O'Donnell HC. et al. (2000). A polipoprotein E genotype and the risk of recurrent lobar intra-cerebral hemorrhage. $N$ Engl J Med. 27.

Reynolds (2003). Alcohol consumption and risk of stroke: a meta-analysis. JAMA: the journal of the American medical association. 289(5).

Schneider et al. (2007). Estimating the burden of disease attributable to alcohol use in South Africa in 2000. South African medical journal. 97.

Sène Diouf, F. et al. (2006). Pronostic fonctionnel des accidents vasculaires cérébraux dans les pays en voie de développement: Sénégal. Ann. Readapt. Med. Phys. 49, Issue 3 :100-104.

Sène Diouf, F. et al. (2008). Survie des accidents vasculaires cérébraux comateux à Dakar (Sénégal). Revue Neurologique.164. Issue 5_: 452-458.

Signorini D. F. ; Andrews PJ. ; Jones PA. ; Wardlaw JM. \& Miller JD. (1999). Adding insult to injury: the prognostic value of early secondary insults for survival after traumatic brain injury. J. Neurol Neurosurg Psychiatry.

Strong et al. (2007). Preventing stroke: saving lives around the word. The lancet neurology. 6(2).

Tossou Kodjo Améwanou. (2014). Facteurs explicatifs de la non application des soins infirmiers à la famille auprès des personnes atteintes d'AVC à l'hôpital central de Yaoundé. Mémoire de Master en Sciences Infirmières. ESS-UCAC.

Toure, K. et al. (2005). Évaluation du coût de prise en charge des accidents vasculaires Cérébraux à Dakar - Sénégal. Médecine tropicale. 65.

Touzé \& Bejot (2009, Chapter 9). Épidémiologie analytique In accidents vasculaires cérébraux. Traité de neurologie.

Wafeu Sadeu, G. (2015). Facteurs déterminants du pronostic vital et fonctionnel des accidents vasculaires cérébraux dans deux hôpitaux universitaires de Yaoundé. The Journal of Medecine and Health Sciences.

Walker, R. et al. (2010). Stroke incidence in rural and urban Tanzania: a prospective, community-based study. The Lancet Neurology. 9. 